\title{
Bone marrow mesenchymal stem cell-derived exosomes attenuate the maturation of dendritic cells and reduce the rejection of allogeneic skin transplantation in mice model
}

\section{RENLI ZHAO}

Southern Medical University https://orcid.org/0000-0002-6039-7447

Guohua Lai

Southern Medical University

Zhiwei Deng

Southern Medical University

\section{Weida Zhuang}

Southern Medical University

Mingjie Wu

Southern Medical University

Jiachang Wu

Southern Medical University

Hongxun Sang ( $\nabla$ hxsang@smu.edu.cn )

Southern Medical University https://orcid.org/0000-0001-6534-0231

\section{Research}

Keywords: bone marrow mesenchymal stem cell, exosomes, dendritic cells, allogenic skin transplant, tolerance

Posted Date: September 15th, 2021

DOI: https://doi.org/10.21203/rs.3.rs-889478/v1

License: (9) This work is licensed under a Creative Commons Attribution 4.0 International License. Read Full License 


\section{Abstract \\ Background}

Bone mesenchymal stem cells (BMSCs)-derived exosomes (B-exos) are attractive for applications in enabling alloantigen tolerance. An in-depth mechanistic understanding of the interaction between B-exos and dendritic cells (DCs) could lead to novel cell-based therapies for allogeneic transplantation. Herein, the potential of a B-exos-based application combined with DCs was explored for inducing allogeneic transplant tolerance.

\section{Methods}

After mixed culture of BMSCs and DCs for 48 hours, DCs from the upper layer were collected to analyze the expression levels of surface markers and mRNAs of inflammation-related cytokines. Then the DCs were co-cultured with B-exo before being collected to detect the mRNA and protein expression levels of indoleamine 2,3-dioxygenase (IDO). The treated DCs from different groups were co-cultured with naïve $\mathrm{CD} 4+\mathrm{T}$ cells from the mouse spleen. The proliferation of CD $4+T$ cells and the proportion of $\mathrm{CD} 4+\mathrm{CD} 25$ + Foxp3 + T cells were analyzed. Finally, the skins of BALB/c mice were transplanted to the back of C57 mice to establish a mouse allogeneic skin transplantation model.

\section{Results}

The coculture of DCs with BMSCs in a trans-well system downregulated the expression of the major histocompatibility complex class II (MHC-II) and CD80/86 costimulatory molecules on DCs, as well as the mRNA expression of interleukin-10 (IL-10), IL-12, and transforming growth factor- $\beta$ (TGF- $\beta$ ). However, these findings were abolished when treated with GW4869. Moreover, B-exos ( $5 \mu \mathrm{g} / \mathrm{mL})$ increased the expression of indoleamine 2,3-dioxygenase (IDO) in DCs treated with lipopolysaccharide (LPS) compared to the control cells. CD4 + CD25 + Foxp3 + T cells increased when cultured with B-exos-exposed DCs, which was attenuated by 1-methyl tryptophan (1MT). Mice recipients injected with B-exos-treated DCs significantly prolonged the skin allograft survival. The allografts showed slight cell infiltration and significantly preserved graft structure. Also, the level of CD $4+C D 25+$ Foxp $3+T$ cells was significantly higher in B-exos-exposed DCs recipient animals than that in other groups.

\section{Conclusions}

Taken together, these data suggested that the B-exos suppress the maturation of DCs and increase the expression of IDO, which might shed light on the role of B-exos in inducing alloantigen tolerance.

\section{Background}


Bone mesenchymal stem cells (BMSCs) have multipotent functions, i.e., forming bone, adipose, and other mesenchymal tissues ${ }^{1}$. In addition to these differentiation capabilities, BMSCs also possess immunomodulatory properties, including the ability to modulate immune cells, such as T, B, natural killer (NK) cells, macrophages, and dendritic cells (DCs) in a non-major histocompatibility complex (MHC)restricted manner both in vitro and in vivo ${ }^{2-4}$. The ability of BMSCs to exert an immunosuppressive effect has been used in the prevention and treatment of graft $v s$. host disease (GVHD) ${ }^{5}$. Several studies indicated that the immunosuppressive effect of MSCs occurs through paracrine or cell-to-cell contact mechanisms ${ }^{6}$.

Exosomes are small membrane vesicles, $40-150 \mathrm{~nm}$, released into the extracellular medium upon fusion of late multivesicular endosomes with the plasma cell membrane ${ }^{7}$ and can transfer the proteins and genetic material to the neighboring cells, leading to varied fate of recipient cells in biological processes ${ }^{8}$. Accumulating evidence has suggested that compared to BMSCs, exosomes have a stable biological activity and a low risk of immunological rejection ${ }^{9}$. Similar to their parent cells, BMSC-derived exosomes (B-exos) mediate immunomodulation by targeting immune cells, such as DCs ${ }^{10}$.

DCs are the most potent antigen-presenting cells (APCs) that play a prominent role in the development of T-cell immune responses ${ }^{11,12}$. In contrast to the mature DCs' (mDCs) ability to stimulate immunity, tolerogenic DCs (tolDCs) are involved in the maintenance of immunological tolerance via T-cell unresponsiveness and generation of regulatory $T$ (Treg) cells ${ }^{13}$. Furthermore, indoleamine 2,3dioxygenase (IDO) downregulates $T$ cell responses by supporting $T$ cell regulatory activity ${ }^{14}$. Another study showed that MSC-exos induced immature DC (imDC) and mDC differentiation into tolerogenic DCs with low expression levels of costimulatory markers in vitro ${ }^{15}$. However, the mechanism underlying $\mathrm{B}$ exos to induce tolDC is yet unknown. Also, whether B-exos from the recipient can drive DCs to become tolDCs and induce transplant immunotolerance is not yet clarified.

Therefore, we investigated whether B-exos exert immunomodulatory effects on DC maturation and function by examining the phenotypic and functional features of B-exos-exposed DCs in comparison to their untreated counterparts, including the expression of maturation/activation markers and the ability to induce Treg cells, as well as the profile of cytokines secreted by DCs. B-exos-treated DCs were further examined for their Treg-inducing ability via the IDO-dependent pathway. We also established an allogenic skin transplant mice model to elucidate the mechanism underlying B-exos-mediated immunomodulation.

\section{Methods}

\section{Animals}

Five-week-old C57BL/ 6 and BALB/c mice (Animal Center of Southern Medical University,Guangzhou[Guangdong), weighing $25-30 \mathrm{~g}$, were utilized. All animal experiments conducted in this study were approved by the Institutional Animal Use Committee of Shenzhen Hospital, Southern 
Medical University, and performed according to the guidelines of the Care and Use of Laboratory Animals (Ministry of Health, China, 1998).

\section{Isolation and culture of BMSCs}

BMSCs were isolated from 5-week-old C57 mouse hind limb bones as described previously ${ }^{16}$ and cultured in MEM-alpha growth medium containing $10 \%$ fetal bovine serum (FBS, Gibco; Thermo Fisher Scientific, Inc.) and $100 \times$ penicillin and streptomycin solution (Gibco). The cells were passaged for $3-5$ passages (P3-5) using $0.25 \%$ trypsin (Gibco) at 78-80\% confluency and used to collect exosomes.

Bovine EV-depleted medium was obtained by overnight ultracentrifugation of medium supplemented with $20 \% \mathrm{FBS}$ at $100,000 \times g\left(4^{\circ} \mathrm{C}\right.$ for $\left.8 \mathrm{~h}\right)$ to eliminate the interference of exosomes from FBS ${ }^{17}$. For exosome isolation, P3-5 BMSCs were washed twice with particle-free Dulbecco's phosphate-buffered saline (DPBS; Sigma-Aldrich, St. Louis, MO, USA) and incubated with fresh medium for continuous culture for $48 \mathrm{~h}$. Subsequently, the culture medium was collected for exosome isolation.

\section{Characterization of BMSCs}

The BMSCs were characterized based on their surface markers and pluripotency differentiation assay. The positive cell surface markers (CD29, CD44, and Sca-1; Biolegend, San Diego, CA, USA) and the negatively expressed markers (CD11b, CD45, and CD19; Biolegend) on cultured BMSCs were evaluated by flow cytometry.

Briefly, $10^{6}$ BMSCs were seeded as a monolayer in six-well plates. OriCell ${ }^{\text {TM }} \mathrm{C} 57$ mouse BMSC adipogenic, osteogenic, and chondrogenic differentiation kits (Catalog no. MUBMX-90031, MUBMX-90021, and MUBMX-9004, respectively) were used according to the manufacturer's instructions (Cyagen Biosciences Inc.). When the adipogenic, osteogenic, and chondrogenic differentiation processes were completed, the cells were fixed with $4 \%$ paraformaldehyde for $30 \mathrm{~min}$, stained with Oil Red 0 working solution, alizarin red, and Alcian blue, respectively, and rinsed with PBS.

\section{Cultivation of BMDCs}

BMDCs were differentiated from bone marrow as described previously ${ }^{18}$. Briefly, bone marrow cells were flushed from the femurs and tibias of 5-week-old C57 mice and cultured in complete RPMI 1640 medium containing 10\% heat-inactivated FBS, $50 \mu \mathrm{M}$ 2-mercaptoethanol, $2 \mathrm{mM}$ glutamine, 100 units $/ \mathrm{mL}$ penicillin, $100 \mu \mathrm{g} / \mathrm{mL}$ streptomycin (Gibco), $20 \mathrm{ng} / \mathrm{mL}$ recombinant mouse granulocyte macrophage colony-stimulating factor (GM-CSF) (Novoprotein, CKO2), and $10 \mathrm{ng} / \mathrm{mL}$ recombinant mouse IL-4 (Novoprotein, CK74). The remaining clusters, loosely adherent to the Petri dish, were cultured at $37^{\circ} \mathrm{C}$ with $5 \% \mathrm{CO}_{2}$, and the medium was changed every alternate day. On day 7 , the cells were collected for treatment and treated with different protocols depending on the subsequent experiments.

\section{Exosome isolation and analysis}


Exosomes were obtained from the supernatant of BMSCs by differential ultracentrifugation as described

previously ${ }^{17}$. Briefly, the pellet was obtained by centrifugation of the culture medium at $300 \times g, 4^{\circ} \mathrm{C}$ for 10 min. The supernatant was clarified as follows: $2000 \times g$ for $20 \mathrm{~min}, 10,000 \times g$ for $40 \mathrm{~min}$, and finally $100,000 \times g$ at $4^{\circ} \mathrm{C}$ for 90 min before resuspension in $50-100 \mu \mathrm{L}$ sterile DPBS.

The ultrastructure and size distribution of exosomes were analyzed by transmission electron microscopy (TEM; Hitachi Ltd) and Nanosight NS300 (Malvern Panalytical). Briefly, the exosome samples were fixed with $1 \%$ glutaraldehyde in PBS at room temperature for $5 \mathrm{~min}$. The mixture was then spotted onto 300-mesh carbon/formvar-coated grids, dried at room temperature, washed with DPBS, and stained for contrast using uranyl acetate $(50 \%)$ in water at room temperature for $10 \mathrm{~min}$. Then, the exosome size and morphology were observed using a JEM-1011 electron microscope (magnification, $\times 25,000$; JEOL Ltd.)

\section{Cellular uptake of exosomes}

Exosomes were labeled with PKH26 using a membrane labeling kit

(Sigma-Aldrich), according to the manufacturer's instruction. Briefly, PKH26 dye was diluted in $100 \mu \mathrm{L}$ diluent $\mathrm{C}$ to a final concentration of $8 \mu \mathrm{M}$ (dye solution). Then, $10 \mu \mathrm{g}$ of exosomes in $20 \mu \mathrm{L}$ DPBS were diluted with $80 \mu \mathrm{L}$ diluent $\mathrm{C}$, added to the dye solution, and incubated for $5 \mathrm{~min}$ after mixing by gentle pipetting. Excess dye was bound with $100 \mu \mathrm{L}$ of 10\% exosome-FBS in RPMI 1640 medium. The exosomes were then diluted to $12 \mathrm{~mL}$ with DPBS and pelleted by ultracentrifugation at $100000 \times \mathrm{g}$ at $4^{\circ} \mathrm{C}$ for $1 \mathrm{~h} 10 \mathrm{~min}$. The pellet was resuspended in $50 \mu \mathrm{L}$ DPBS. ImDCs were incubated with $5 \mu \mathrm{g} / \mathrm{mL}$ of PKH26-labeled exosomes for $12 \mathrm{~h}$ and stained with DiO (Beyotime, C1993S) and DAPI (Beyotime, C1005) before being observed using a Benchtop High-Content Analysis System (Yokogawa, CQ1).

\section{Flow cytometry analysis}

P3 BMSCs were digested with $0.25 \%$ trypsin-EDTA and suspended to a concentration of $1 \times 10^{6} \mathrm{cells} / \mathrm{mL}$ in cell staining buffer (Biolegend, \#156603). Next, the cell suspension was stained with antibodies from Biolegend against CD29-PE (\#102207), CD44-PE (\#103023), Sca-1-PE (\#108107), CD11b-PE (\#101207), CD45-PE (\#157603), and CD19-PE (\#152407) in the dark at $4^{\circ} \mathrm{C}$ for $45 \mathrm{~min}$.

Immature BMDCs were cultivated in six-well plates at $5 \times 10^{6}$ cells/well. BMDCs were left untreated (as a control) or stimulated with $1 \mathrm{mg} / \mathrm{mL}$ lipopolysaccharide (LPS), $5 \mu \mathrm{g} / \mathrm{mL}$ of BMSC-exos or $1 \mathrm{mM} 1$-methylL-tryptophan (1MT, Sigma-Aldrich) for $48 \mathrm{~h}$. BMDCs in different groups were then incubated with the following anti-mouse antibodies (Biolegend): FITC-conjugated anti-CD11c (\#117305) and PE-conjugated anti-CD80 (\#104707), -CD86 (\#159203), and -MHC II (\#116407). Subsequently, the cells were stained and observed using a Fortessa flow cytometer (BD Biosciences), and data were analyzed with FlowJo V10 software (FlowJo, LLC).

Intracellular staining was performed with a Foxp3/Transcription Factor Staining Buffer Set (Liankebio, KTR201-100). Tregs were stained with FITC-conjugated anti-mouse CD4 (GK1.5), APC-conjugated anti- 
mouse CD25 (PC61.5), and PE-conjugated anti-mouse Foxp3 Ab (3G3) mAbs. The populations of Tregs in the cocultured cell samples were defined as CD4 + CD25 + Foxp3+.

\section{Coculture of BMSCs and DCs in a transwell system}

To confirm that the B-exos attenuate the maturation of dendritic cells, BMSCs were cultured in complete medium in the lower well of trans-well chambers (pore size, 0,4 $\mathrm{mm}$, Costar Corning) to $80 \%$ confluency. Then, the medium was replaced with a complete RPMI 1640 medium containing 10\% exosome-depleted FBS. For inhibition of exosome generation, BMSCs were incubated with $20 \mu \mathrm{M} \mathrm{GW} 4869^{19}$ (Sigma) for 24 h, and the medium was replaced with RPMI 1640 containing 10\% exosome-depleted FBS before coculture. Subsequently, BMSCs were cultured in the lower compartment, while DCs were cultured in the upper compartment to avoid cell-cell contact. DCs were harvested for further experiments after coculturing in the transwell system for $48 \mathrm{~h}$.

\section{Western blot}

Proteins were extracted from the isolated exosomes or DCs from differentially treated groups using the Whole Cell Lysis Assay (KeyGen Biotech, Nanjing, China), and the concentration was determined using the FD ${ }^{\text {TM }}$ BCA Protein Quantitative Kit (Fdbio Science, Hangzhou, China). The lysates were resolved by 10\% SDS-PAGE, and the separated proteins were transferred to PVDF membranes (EMD Millipore).

After blocking with 5\% skimmed milk in TBST buffer at room temperature for $1 \mathrm{~h}$, the membranes were probed with the following primary antibodies at $4^{\circ} \mathrm{C}$ overnight (Abcam): TSG101 (1:1000, ab125011), CD81 (1:1000, ab109201), CD9 (1:2000, ab92726), CD63 (1:1000, ab217345), and IDO (1:1000, ab277522). Subsequently, the membranes were incubated with horseradish peroxidase (HRP)-coupled goat anti-rabbit IgG H\&L (1:10,000, Abcam, ab205718) and developed using BeyoECL Star (Beyotime, Shanghai, China). The intensity of the immunoreactive bands was analyzed using the ChemiDoc Imaging System (Bio-Rad).

\section{Coculture of DCs and naïve CD4 + T cells for differentiation of Tregs}

Naive CD4 + T cells were purified from pooled single-cell suspensions of C57 spleen using a mouse naive CD4 + T cell isolation kit (Biolegend, \#480039). The purity of the enriched subset was validated by flow cytometry and was found to be $>95 \%$. These purified naive CD $4+T$ cells were cocultured with DC s from differentially treated groups at an optimal ratio described previously ${ }^{20}$.Then, $1 \mathrm{mM} 1$-methyl tryptophan (1MT), a selective IDO inhibitor, was added to the medium in the 1MT group before coculture. The cells (T cell $10^{6}$ and DCs $2 \times 10^{5}$ ) were cultured in 24-well plates with a total volume of $1 \mathrm{~mL} /$ well of culture medium. The medium was refreshed on days 3 and 5 , and the cells were harvested for flow cytometry analysis on Day 5.

\section{Real-time quantitative polymerase chain reaction (RT-qPCR)}


Total RNA was isolated from dendritic cells in different groups using TRIzol® (Thermo Fisher Scientific) following the manufacturer's protocols. RNA quantity and quality were measured on a NanoDrop ND-1000 spectrophotometer (NanoDrop Technologies; Thermo Fisher Scientific). An equivalent of $1 \mu \mathrm{g}$ RNA was reverse-transcribed using a reverse transcription kit (TransGen Biotech Co., Ltd). RT-qPCR was performed using a PerfectStart Green qPCR SuperMix kit (TransGen Biotech Co., Ltd) on an ABI-7500 machine (Applied Biosystems; Thermo Fisher Scientific, Inc.). The primer pairs are listed in Table 1. The data were analyzed using SDS relative quantification software (version 2.2.2; Thermo Fisher Scientific, Inc.). The relative fold-change was calculated using the $2^{-\Delta \Delta C t}$ method ${ }^{21}$. All reactions were performed in triplicate and normalized to the expression of the internal control Actin.

\section{Allogenic skin transplant}

Full-thickness skin transplantation in mice was performed as described previously ${ }^{22}$. Briefly, the donor mouse (BALB/C) was anesthetized, and the back skin from the hip to the neck was harvested and cut into $15-\mathrm{mm} \times 15-\mathrm{mm}$ grafts. For the recipient (C57BL/6), $5 \times 10^{6}$ dendritic cells were given via tail vein on postoperation day (POD) 0. A 10-mm×10-mm to $15-\mathrm{mm} \times 15-\mathrm{mm}$ square of skin was cut superficially after anesthesia. The graft from the donor was positioned on the graft bed, and eight sutures were placed on the corners and the middle of each edge. Finally, the recipient mouse was wrapped in an adhesive bandage with folded gauze over the graft. Seven days post-surgery, the bandage was removed by cutting only through the ventral side of the covering. Signs of rejection were monitored daily. On POD12, skin and spleen samples were harvested for analysis.

\section{Histopathology}

On POD12, skin samples were collected, and animals were euthanized by $\mathrm{CO}_{2}$ inhalation. The samples were fixed in $10 \%$ neutral-buffered formalin, processed in graded alcohols, sectioned into 5 - $\mu \mathrm{m}$-thick slices, and stained with hematoxylin and eosin (H\&E). All slides were reviewed by an expert veterinary pathologist (SEB) in a blinded manner.

\section{Statistical analysis}

The data are expressed as the means \pm standard deviations. Statistical analyses were performed using a one-way analysis of variance (ANOVA) combined with Dunnett's t-test. Graft survival was compared by Kaplan-Meier analysis and the log-rank test. $\mathrm{P}<0.05$ indicated statistical significance.

\section{Results}

\section{Identification of BMSCs and B-exos}

BMSCs from C57 mice could be induced towards osteogenic and lipogenic chondrogenic differentiation (Fig. 1A-C). The results of flow cytometry verified that the cells used in our experiment expressed identical BMSC markers in line with the definition of BMSCs (Fig. 1D). 
Then, exosomes were isolated from the supernatant of P3-5 BMSCs according to the protocol in Supplementary Fig. 1A. TEM, nanoparticle tracking analysis (NTA), and western blot analysis were used to analyze the ultrastructure, size distribution, and specific protein, respectively. The results showed that the exosome size was about $89 \mathrm{~nm}$ (Fig. 2A), and the characteristic saucer shape was revealed by TEM (Fig. 2B). Additionally, the expression levels of CD9, CD63, CD81, and TSG101 were more abundant in the exosome protein lysis compared to their parental cell protein lysis (Fig. 2C). These findings indicated the successful isolation of BMSCs and exosomes.

\section{Exosome uptake and effects on attenuation of dendritic cells maturation}

To assess if B-exos interact directly with dendritic cells from C57 mice, imDCs differentiated from bone marrow were incubated with PKH26-labeled exosomes and monitored by fluorescence microscopy imaging over $12 \mathrm{~h}$. We observed that the cells endocytosed the exosomes and became fluorescent at $12 \mathrm{~h}$ (Fig. 3A). These findings implied that B-exos have the potential to communicate directly with allogenic imDCs.

Next, we investigated whether B-exos affect the phenotype and function of mDCs through indirect contact with BMSCs in vitro in trans-well chambers (Fig. 3B). The median fluorescence intensity (MFI) shift showed a significant decrease in MHCll, CD86, and CD80 in DCs cocultured with BMSCs compared to that of mDCs; however, these effects were abolished when treated with BMSCs treated with GW4869, the exosome inhibitor (Fig. 3B-C).

Then, we performed qRT-PCR-based immune profiling of DCs from different groups. The BMSC-exposed DCs showed significantly increased transcripts of several immune-modulatory genes, including $I L-10$, IL12 , and $T G F-\beta$, whereas transcripts of $I L-27$ and $I L-6$ were decreased compared to mDCs. However, these phenomena were abolished post-treatment with GW4869 (Fig. 4).

\section{IDO is increased during B-exos-induced differentiation of dendritic cells}

Since BMSC-exposed mDCs increased IDO expression, we investigated the involvement of IDO in the induction of tolDCs by BMSCs-exos. The exosomes were purified from BMSCs supernatants and coincubated with imDCs treated with or without LPS $(5 \mu \mathrm{g} / \mathrm{mL})$ for $48 \mathrm{~h}$. Then, we analyzed the effects of different doses $(1,2,5,8$, or $10 \mu \mathrm{g} / \mathrm{mL})$ of BMSCs-exos. Surprisingly, we found that the expression levels of IDO in mDCs were increased in 2,5 , and $10 \mu \mathrm{g} / \mathrm{mL}$ groups compared to the mDCs group, and the highest expression was obtained in the $5 \mu \mathrm{g} / \mathrm{mL}$ group (Fig. $5 A-B$ ), deeming it as the optimal dosage for increasing IDO expression.

\section{Effect of IDO on B-exos-exposed DCs}


We also investigated if B-exos-treated DCs could induce Treg polarization. Naïve CD $4+T$ cells $\left(1 \times 10^{6} /\right.$ well) were isolated from the spleen and incubated with DCs $\left(2.5 \times 10^{5} /\right.$ well $)$ in different groups for 5 days. As shown in Fig. 5C-D, CD $4+C D 25+$ Foxp3 + T cells decreased by mDCs and IMT-treated mDCs but increased in B-exos-exposed DCs. These findings demonstrated that B-exos-exposed DCs induced regs by increasing IDO.

\section{B-exos-exposed DCs enhanced allogeneic skin graft}

Based on the enhancement of Treg polarization by B-exos-exposed DCs, we hypothesized that B-exos exposed DCs could delay the allogeneic skin graft rejection with a concomitant increase in Tregs in the recipient mice. The back skins from BALB/c mice were grafted on BALB/c recipients and followed by caudal vein injections of $5 \times 10^{5}$ DCs per mouse on POD0 (Fig. 6A).

Recipient mice injected with DCs and DCs in the 1MT group rejected allograft skins (median survival time [MSTs]: day14 and day13, respectively; Fig. 6B), whereas those injected with B-exos-exposed DCs had significantly prolonged allograft survival (MST: 18 days). Therefore, the DCs in B-exos group administration significantly improved the skin allograft survival.

Histological examination of skin allografts showed slight cell inf tration and preserved graft structure in transplant recipients injected with B-exos-exposed DCs on POD12. However, allografts from recipients injected with $\mathrm{mDCs}$ and DCs in the $1 \mathrm{MT}$ group showed severe myocyte damage and moderate inflammatory infiltration (Fig. 6E).

\section{B-exos-exposed DCs induced the proliferation of recipient spleen CD4 + CD25 + Foxp3 + T cells}

To determine if this delayed graft rejection was due to increased Treg polarization, the spleens of the mice from different groups were harvested on day 12 and assayed for Tregs. The level of CD4 + CD25+ Foxp3 $+T$ cells was significantly higher in B-exos-exposed DC recipient animals compared to that of $\mathrm{mDCs}$ and DCs in $1 \mathrm{MT}$ group (Fig. 7A-B).

\section{Discussion}

Due to their potential applications in enabling alloantigen tolerance, MSCs have been investigated thoroughly and found to exert an immunomodulatory effect that influences all cells involved in the immune response ${ }^{23}$. Moreover, the paracrine effect is one of the critical mechanisms of MSCs underlying immune tolerance ${ }^{24}$. Bruno et al. ${ }^{25}$ demonstrated that exosomes play a significant role in the paracrine effect of MSCs. As a tool of cell-to-cell communication, exosomes transfer biological material between the cells in the form of paracrine and remote secretion and regulate the physiological and pathological conditions $^{23}$. Several studies have shown that MSC-derived exosomes are enabled to affect the activity of immune cells, including T cells, B cells, NK cells, and macrophages. A recent study by Zhang et al. ${ }^{26}$ showed that MSC exosomes mediated cartilage repair by enhancing proliferation, attenuating apoptosis, 
and modulating immune reactivity by inducing M2 macrophages and reducing pro-inflammatory synovial cytokines. Furthermore, murine adipose-derived MSCs (AD-MSCs) induced tolDCs with low maturation and expression of surface markers and high production of immunomodulatory cytokines ${ }^{15}$. Similar results were demonstrated by Monica et al. ${ }^{10}$, showing that MSC-EV treatment impaired antigen uptake by imDCs and halted DC maturation. These findings indicated that the regenerative and immunomodulatory properties of MSCs were inherited by MSC-exos, which are convenient and recommended for alternative treatments ${ }^{27}$.

In agreement with previous studies, we found that B-exos can attenuate CD $11^{+}$DCs maturation. In this study, an indirect cell-to-cell contact trans-well system was established to investigate the inhibition effect of B-exos on DCs. Cocultured with BMSCs, imDCs differentiated into a tolerance phenotype in the presence of LPS and exhibited a low expression of MHCII, CD86, and CD80. In addition, BMSCs affect the release of $T G F-\beta, I L-10, I L-12, I L-6, I L-27$, and INF- $\beta$ from DCs and enhance the ability of DCs to induce Tregs. Then, GW4869, an inhibitor of exosome secretion, was added to inhibit BMSCs-exos production. These data suggested that when B-exos were inhibited by GW4869, the function of BMSCs above was partly reversed. Taking together the results from rescue experiments, we concluded that BMSCs-exos, as a form of remote secretion by BMSCs, attenuate the maturation and activation of DCs and induce mDCs into tolDCs.

DCs are essential in directing immune responses toward either immunity or tolerance ${ }^{28}$. TolDCs are a subset of DCs that can induce tolerance through various mechanisms, including the induction of Tregs, autoreactive T cell anergy, and apoptosis, and could be used in tolerizing immunotherapies ${ }^{29}$. Phase I and II clinical trials utilizing toIDCs have been conducted for kidney and liver transplant recipients ${ }^{30}$. A key mechanism involved in tolDCs-mediated immunosuppression is the expression of IDO- ${ }^{31}$. In addition to suppression of proliferation, IDO competence in human DCs is shown to support T cell regulatory function ${ }^{32,33}$. The current data suggested that when exposed to BMSCs-exos, DCs increase the expression of IDO in a dose-dependent manner. CD4 + CD25 + Foxp3 + T-cells were increased in the BMSCexos-exposed DC group compared to those in the non-exposed DC group. However, this tendency was reversed by $1 \mathrm{MT}$, a selective IDO inhibitor. Collectively, BMSC-exos enhanced the ability of DCs to induce Tregs via elevated expression of IDO. These results confirmed the findings of a previous study, wherein MSC exosomes required monocytes to mediate differentiation of CD4 + T cells to Tregs ${ }^{34}$.

Finally, a mouse skin transplant model was used to study the immunoregulatory properties of B-exosexposed DCs. Allograft tolerance was induced by B-exos-exposed DCs with a prolonged skin allograft survival. The histological results showed slight cell infiltration and significantly preserved graft structure in B-exos-exposed DC injection group. Furthermore, the level of CD4 + CD25 + Foxp3 + T cells was significantly higher in the spleen of B-exos-exposed DCs recipient animals. Presently, the clinical application of MSCs in organ transplantation used to improve the prognosis of transplant recipients has broad prospects. Although MSC-derived exosomes have functions similar to MSCs, the direct application is not yet mature. Thus, in the present study, DCs were chosen as recipient cells to modulate allograft 
tolerance. The regulatory DCs promote liver transplant operational tolerance and are used in cell therapy for many autoimmune disease ${ }^{35,36}$. However, our cell injection strategy could not achieve long-term transplant survival. Notably, it is difficult to achieve tolerance or long-term survival without the use of immunosuppressants ${ }^{23}$. However, tolDCs combined with suboptimal doses of immunosuppressants may achieve specific allograft tolerance and long-term transplant survival ${ }^{37}$. Another reason for short-term survival is that we only injected DCs on POD0; post-operative, it might show an improved allograft tolerance.

Furthermore, the present study showed that B-exos induce tolDCs and increase the expression of IDO, but the exact protein or non-coding RNA constituent that promotes this effect is yet to be identified. As a cargo for cell-to-cell communication, BMSC-exos transfer bioactive molecules to DCs are the key factors for future studies. The critical factors involved in the induction of tolDCs may be illustrated by RNA sequencing and proteomic analysis along with CRISPR/Cas9 deletion screening or antibody blocking studies. Next, a multiple injection strategy of tolDCs combined with suboptimal doses of immunosuppressants might achieve specific allograft tolerance and long-term transplant survival.

\section{Conclusion}

In conclusion, the present in vivo and in vitro study showed that B-exos induce mDCs into a tolerogenic DCs population with low expression of costimulatory markers and high IDO. These tolerogenic DCs can induce Tregs, increase the secretion of $I L-10, I L-12$, and $T G F-\beta$, and decrease secretion of $I L-6$ and $I L-27$. In the in vivo study, allograft tolerance is induced by B-exos-exposed DCs with prolonged skin allograft survival.

\section{Abbreviations}

\section{Bexo}

Bone mesenchymal stem cells-derived exosomes

\section{BMSCs}

Bone mesenchymal stem cells

\section{DCs}

Dendritic cells

imDCs

Immature dendritic cells

\section{mDCs}

Mature dendritic cells

tolDCs

Tolerogenic dendritic cells

\section{LPS}

Lipopolysaccharide

IDO 
Indoleamine 2,3-dioxygenase

MHC-II

Major histocompatibility complex class II

IL

Interleukin

TGF- $\beta$

Transforming growth factor- $\beta$

1MT

1-methyl tryptophan

PBS

Phosphate buffered saline

\section{GM-CSF}

Granulocyte-macrophage colony-stimulating factor

MFI

Median Fluorescence Intensity

DMSO

Dimethyl sulfoxide

\section{Declarations}

\section{Ethics approval and consent to participate}

Not applicable.

\section{Consent for publication}

Not applicable.

\section{Availability of data and materials}

We confirming absence of shared data in this paper.

\section{Competing interests}

The authors declare that there is no conflict of interests.

\section{Funding}

This study was supported by National Natural Science Foundation of China (81871767), Sanming Project of Medicine in Shenzhen (SZSM201612019), Shenzhen Key Laboratory of Digital Surgical Printing Project (ZDSYS201707311542415), Southern Medical University Clinical Project (LC2016ZD036), Shenzhen Fundamental Research Key Project (JCYJ20200109150641992) and the Key-Area Research and Development Program of Guangdong Province, China (2020B010165004). 


\section{Authors' contributions}

Hongxun Sang conceived the project and developed the experimental design. Renli Zhao, Guohua Lai and Zhiwei Deng participated in the collection and assembly of data, data analysis and interpretation. Weida Zhuang and Mingjie Wu analyzed data and revised the manuscript, and Jiachang Wu participated in the design and supervision of research. Renli Zhao wrote the paper. Hongxun Sang edited the manuscript. All authors reviewed the manuscript.

\section{Acknowledgements}

Not applicable.

\section{References}

1. Pittenger MF, et al. Multilineage potential of adult human mesenchymal stem cells. Science. 1999;284:143-7. doi:10.1126/science.284.5411.143.

2. Le Blanc K. Immunomodulatory effects of fetal and adult mesenchymal stem cells. Cytotherapy. 2003;5:485-9. doi:10.1080/14653240310003611.

3. Li X, et al. Mesenchymal stem cells induced regulatory dendritic cells from hemopoietic progenitor cells through Notch pathway and TGF- $\beta$ synergistically. Immunology letters. 2020;222:49-57. doi:10.1016/j.imlet.2020.03.005.

4. Palomares Cabeza V, et al. Pediatric Mesenchymal Stem Cells Exhibit Immunomodulatory Properties Toward Allogeneic T and B Cells Under Inflammatory Conditions. Front Bioeng Biotechnol. 2019;7:142. doi:10.3389/fbioe.2019.00142.

5. Le Blanc K, et al. Treatment of severe acute graft-versus-host disease with third party haploidentical mesenchymal stem cells. Lancet. 2004;363:1439-41. doi:10.1016/s0140-6736(04)16104-7.

6. Li YP, et al. Human mesenchymal stem cells license adult CD34 + hemopoietic progenitor cells to differentiate into regulatory dendritic cells through activation of the Notch pathway. Journal of immunology (Baltimore Md: 1950). 2008;180:1598-608. doi:10.4049/jimmunol.180.3.1598.

7. Escola JM, et al. Selective enrichment of tetraspan proteins on the internal vesicles of multivesicular endosomes and on exosomes secreted by human B-lymphocytes. J Biol Chem. 1998;273:20121-7. doi:10.1074/jbc.273.32.20121.

8. Huang Y, Liu K, Li Q, Yao Y, Wang Y. Exosomes Function in Tumor Immune Microenvironment. Adv Exp Med Biol. 2018;1056:109-22. doi:10.1007/978-3-319-74470-4_7.

9. Raposo G, Stoorvogel W. Extracellular vesicles: exosomes, microvesicles, and friends. J Cell Biol. 2013;200:373-83. doi:10.1083/jcb.201211138.

10. Reis M, et al. Mesenchymal Stromal Cell-Derived Extracellular Vesicles Attenuate Dendritic Cell Maturation and Function. Frontiers in immunology. 2018;9:2538. doi:10.3389/fimmu.2018.02538. 
11. Banchereau J, Steinman RM. Dendritic cells and the control of immunity. Nature. 1998;392:245-52. doi:10.1038/32588.

12. Lanzavecchia A, Sallusto F. Regulation of T cell immunity by dendritic cells. Cell. 2001;106:263-6. doi:10.1016/s0092-8674(01)00455-x.

13. Yoo S, Ha SJ. Generation of Tolerogenic Dendritic Cells and Their Therapeutic Applications. Immune network. 2016;16:52-60. doi:10.4110/in.2016.16.1.52.

14. Heitger A. Regulation of expression and function of IDO in human dendritic cells. Curr Med Chem. 2011;18:2222-33. doi:10.2174/092986711795656018.

15. Shahir M, et al. Effect of mesenchymal stem cell-derived exosomes on the induction of mouse tolerogenic dendritic cells. Journal of cellular physiology. 2020;235:7043-55. doi:10.1002/jcp.29601.

16. Soleimani M, Nadri S. A protocol for isolation and culture of mesenchymal stem cells from mouse bone marrow. Nature protocols. 2009;4:102-6. doi:10.1038/nprot.2008.221.

17. Kowal J, et al. Proteomic comparison defines novel markers to characterize heterogeneous populations of extracellular vesicle subtypes. Proc Natl Acad Sci USA. 2016;113:E968-77. doi:10.1073/pnas.1521230113.

18. Wang W, Li J, Wu K, Azhati B, Rexiati M. Culture and Identification of Mouse Bone Marrow-Derived Dendritic Cells and Their Capability to Induce T Lymphocyte Proliferation. Med Sci Monit. 2016;22:244-50. doi:10.12659/msm.896951.

19. Xiao C, et al. Transplanted Mesenchymal Stem Cells Reduce Autophagic Flux in Infarcted Hearts via the Exosomal Transfer of miR-125b. Circulation research. 2018;123:564-78. doi:10.1161/circresaha.118.312758.

20. Jain $A$, et al. T cells instruct myeloid cells to produce inflammasome-independent IL-1 $\beta$ and cause autoimmunity. Nature immunology. 2020;21:65-74. doi:10.1038/s41590-019-0559-y.

21. Livak KJ, Schmittgen TD. Analysis of relative gene expression data using real-time quantitative PCR and the 2(-Delta Delta C(T)) Method. Methods. 2001;25:402-8. doi:10.1006/meth.2001.1262.

22. Cheng $\mathrm{CH}$, et al. Murine Full-thickness Skin Transplantation. Journal of visualized experiments: JoVE. 2017. doi:10.3791/55105.

23. Zheng Q, Zhang S, Guo WZ, Li XK. The Unique Immunomodulatory Properties of MSC-Derived Exosomes in Organ Transplantation. Frontiers in immunology. 2021;12:659621. doi:10.3389/fimmu.2021.659621.

24. Spees JL, Lee RH, Gregory CA. Mechanisms of mesenchymal stem/stromal cell function. Stem Cell Res Ther. 2016;7:125. doi:10.1186/s13287-016-0363-7.

25. Bruno S, Kholia S, Deregibus MC, Camussi G. The Role of Extracellular Vesicles as Paracrine Effectors in Stem Cell-Based Therapies. Adv Exp Med Biol. 2019;1201:175-93. doi:10.1007/978-3030-31206-0_9. 
26. Zhang $\mathrm{S}$, et al. MSC exosomes mediate cartilage repair by enhancing proliferation, attenuating apoptosis and modulating immune reactivity. Biomaterials. 2018;156:16-27. doi:10.1016/j.biomaterials.2017.11.028.

27. Askenase PW. COVID-19 therapy with mesenchymal stromal cells (MSC) and convalescent plasma must consider exosome involvement: Do the exosomes in convalescent plasma antagonize the weak immune antibodies? Journal of extracellular vesicles. 2020;10:e12004. doi:10.1002/jev2.12004.

28. Castenmiller C, Keumatio-Doungtsop BC, van Ree R, de Jong EC, van Kooyk Y. Tolerogenic Immunotherapy: Targeting DC Surface Receptors to Induce Antigen-Specific Tolerance. Frontiers in immunology. 2021;12:643240. doi:10.3389/fimmu.2021.643240.

29. Obregon C, Kumar R, Pascual MA, Vassalli G, Golshayan D. Update on Dendritic Cell-Induced Immunological and Clinical Tolerance. Frontiers in immunology. 2017;8:1514. doi:10.3389/fimmu.2017.01514.

30. Ten Brinke A, et al. Ways Forward for Tolerance-Inducing Cellular Therapies- an AFACTT Perspective. Frontiers in immunology. 2019;10:181. doi:10.3389/fimmu.2019.00181.

31. Mellor AL, Lemos H, Huang L. Indoleamine 2,3-Dioxygenase and Tolerance: Where Are We Now? Frontiers in immunology. 2017;8:1360. doi:10.3389/fimmu.2017.01360.

32. Chen W, Liang X, Peterson AJ, Munn DH, Blazar BR. The indoleamine 2,3-dioxygenase pathway is essential for human plasmacytoid dendritic cell-induced adaptive $T$ regulatory cell generation. Journal of immunology (Baltimore Md: 1950). 2008;181:5396-404. doi:10.4049/jimmunol.181.8.5396.

33. Chung DJ, et al. Indoleamine 2,3-dioxygenase-expressing mature human monocyte-derived dendritic cells expand potent autologous regulatory T cells. Blood. 2009;114:555-63. doi:10.1182/blood2008-11-191197.

34. Zhang B, et al. Mesenchymal stem cells secrete immunologically active exosomes. Stem Cells Dev. 2014;23:1233-44. doi:10.1089/scd.2013.0479.

35. Thomson AW, Humar A, Lakkis FG, Metes DM. Regulatory dendritic cells for promotion of liver transplant operational tolerance: Rationale for a clinical trial and accompanying mechanistic studies. Hum Immunol. 2018;79:314-21. doi:10.1016/j.humimm.2017.10.017.

36. Li D, et al. Transplantation of Aire-overexpressing bone marrow-derived dendritic cells delays the onset of type 1 diabetes. Int Immunopharmacol. 2017;49:13-20. doi:10.1016/j.intimp.2017.05.023.

37. Monguió-Tortajada M, Lauzurica-Valdemoros R, Borràs FE. Tolerance in organ transplantation: from conventional immunosuppression to extracellular vesicles. Frontiers in immunology. 2014;5:416. doi:10.3389/fimmu.2014.00416.

\section{Tables}

The primer pairs are listed in Table 1 


\begin{tabular}{|lll|}
\hline Gene & Sequence & Source \\
\hline TGF- $\beta$ F & TGTCACAACTCAGCCAACAGG & Sangon Biotech \\
\hline TGF- $\beta$ R & CAACCAGCCTCCTAAACACCC & Sangon Biotech \\
\hline IL10 F & CAGAGCCACATGCTCCTAGA & Sangon Biotech \\
\hline IL12b F & GACTCCAGGGGACAGGCTA & Sangon Biotech \\
\hline IL12b R & CCAGGAGATGGTTAGCTTCTGA & Sangon Biotech \\
\hline IL6F & GCTACCAAACTGGATATAATCAGGA & Sangon Biotech \\
\hline IL6 R & CCAGGTAGCTATGGTACTCCAGAA & Sangon Biotech \\
\hline IL27_F & GCAGGGAATTCACAGTCAGC & Sangon Biotech \\
\hline IL27_R & GGACATAGCCCTGAACCTCA & Sangon Biotech \\
\hline ID01_F & GGGCTTTGCTCTACCACATC & Sangon Biotech \\
\hline ID01_R & AAGGACCCAGGGGCTGTAT & Sangon Biotech \\
\hline Actin F & GGCTGTATTCCCCTCCATCG & Sangon Biotech \\
\hline Actin R & CCAGTTGGTAACAATGCCATG & Sangon Biotech \\
\hline
\end{tabular}

\section{Figures}

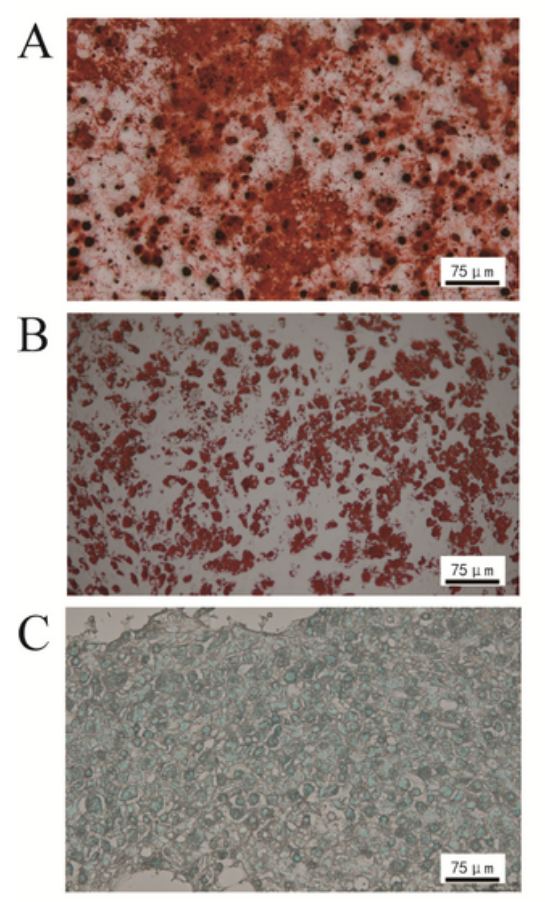

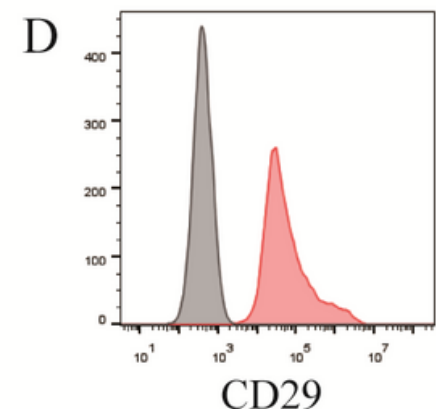

CD29

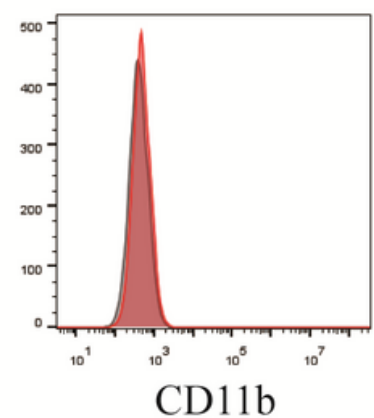

CD11b
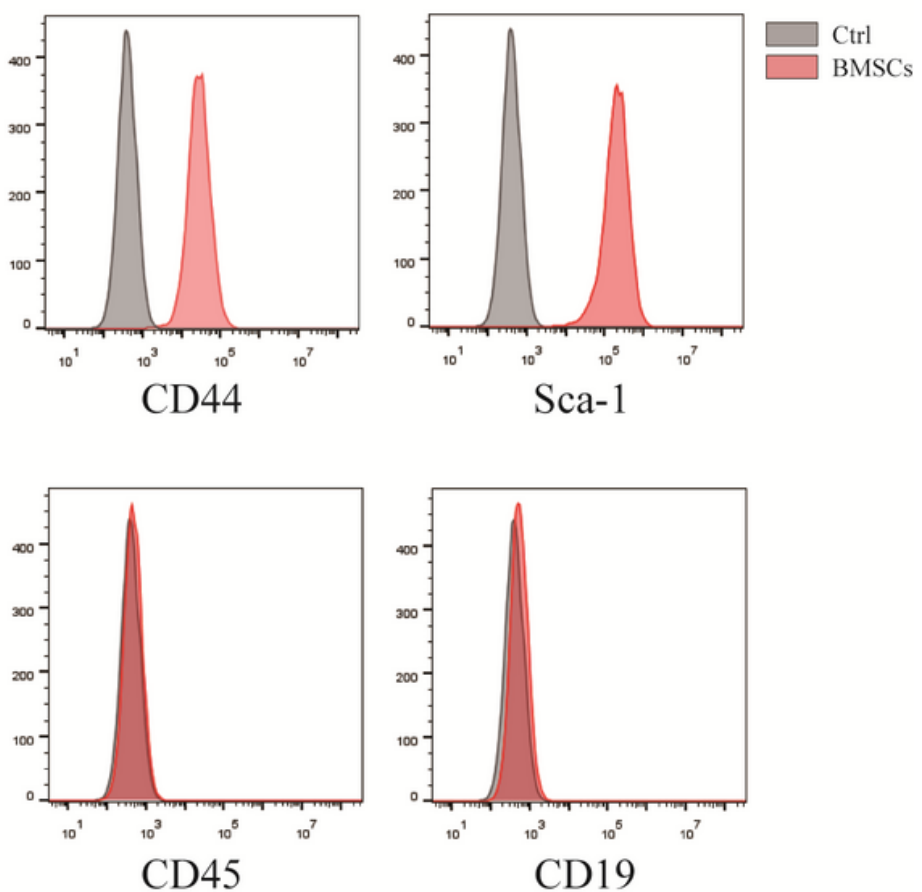
Figure 1

Results of BMSCs identification. A: After 28 days Osteogenic induction, calcium nodules could be seen under (400X) microscope stained by alizarin red. B: After 28 days adipogenic induction, red lipid droplets could be seen under (400X) microscope stained by oil red O. C: After 28 days chondrogenic induction, Blue endoacidic mucopolysaccharides could be seen under (400X) microscope stained by Alcian blue. D: Flow cytometry results showed that CD29, CD44 and Sca-1 were expressed on the surface of BMSCs, but CD11b, CD45 and CD19 were not.

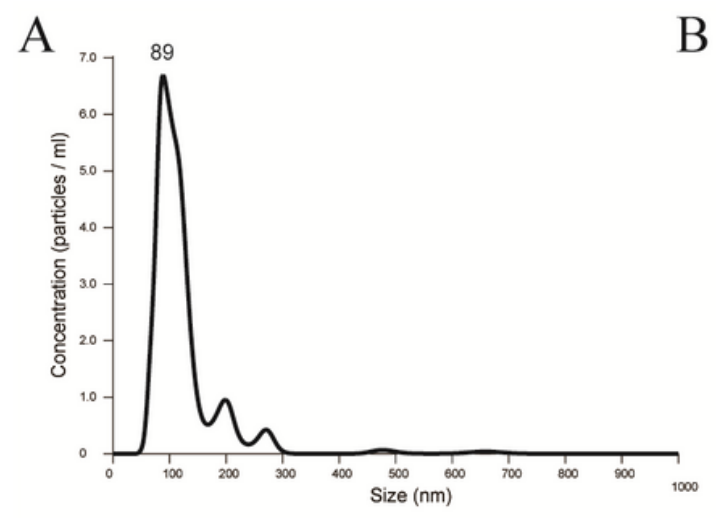

B

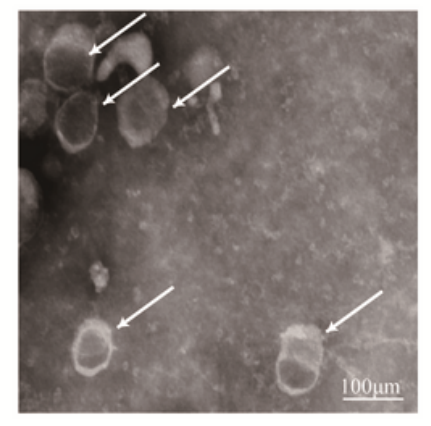

C

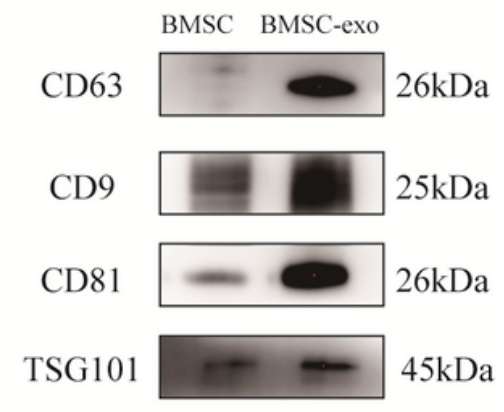

Figure 2

Identification of BMSCs-derived exosomes. A: TEM results revealed the characteristic saucer shape of exosomes (white arrows). C: Nanoparticle Tracking Analysis demonstrated that the B-exo had a mean particle diameter of $107 \mathrm{~nm}$. D: CD9, CD63, CD81 and TSG101 were more abundant in the exosome protein lysis compared with BMSCs protein lysis $₫ A$ total of $5 \mu \mathrm{g}$ protein from BMSCs lysis and $5 \mu \mathrm{g}$ protein from exosomes lysis were tested》. 


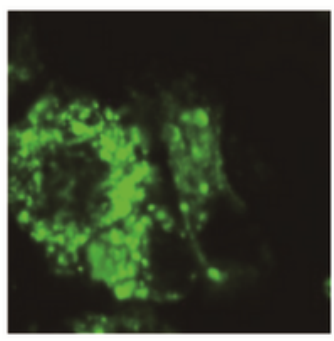

imDC-DiO

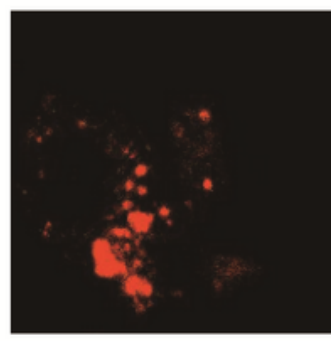

B-exo-PKH26

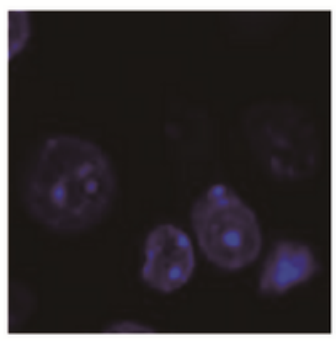

DAPI

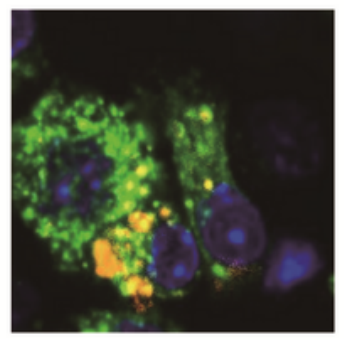

Merge

A

Trans-well

Day6 imDCs

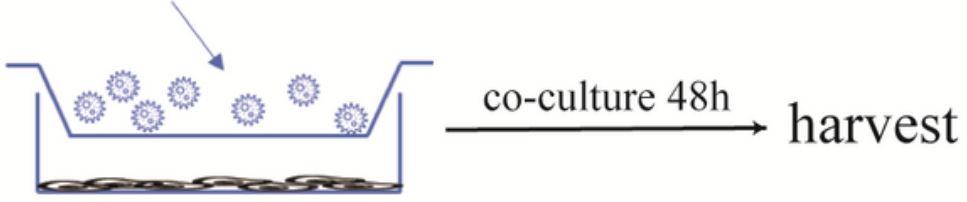

BMSCs

B

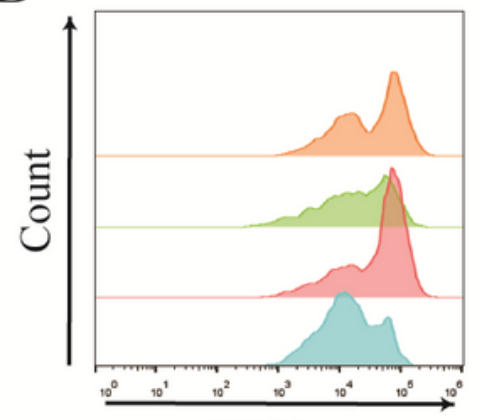

CD80

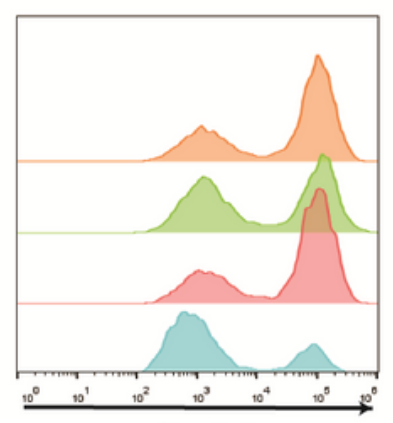

CD86

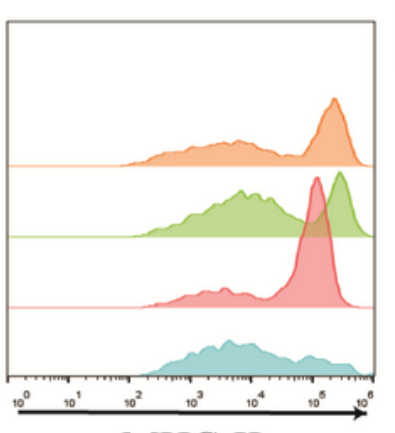

MHC-II
imDC+LPS+BMSCs+GW4869 imDC+LPS+BMSCs imDC + LPS imDC

C
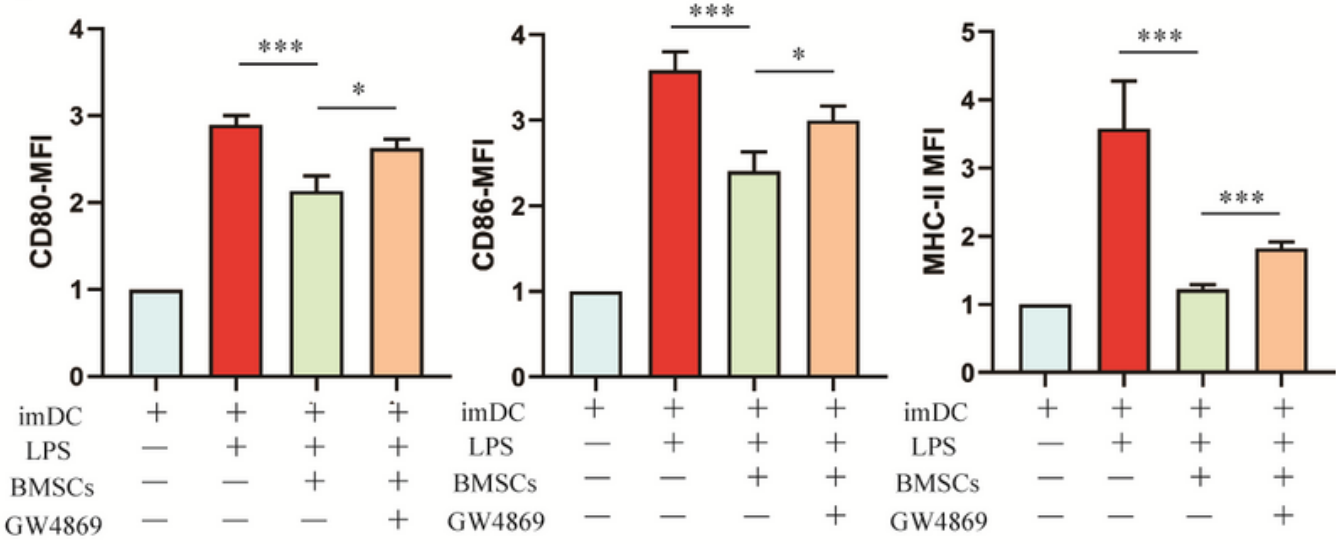

Figure 3

Exosome uptake and effects on attenuation of dendritic cells maturation. A: DCs uptake assay by confocal microscopy demonstrated rapid uptake of B-exo (green: imDC membrane-DiO; red: exosomePKH26; blue: cell nucleus-DAPI). B: Flow chart of Trans-well co-culture system. C: DC cells were gated by CD11C+ CD80+, CD11 + CD86+ and CD11 c+ MHC-II+ respectively, then based on these gates, histograms were drawn up. Representative histogram shows the expression of CD80, CD86 and MHC-II. D: Bar graphs 
represent mean fluorescence intensity fold change \pm SEM of CD80, CD86 and MHCII in the various groups from $\mathrm{n}=3$ independent experiments. ${ }^{\star} \mathrm{P}<0.05,{ }^{\star *} \mathrm{P}<0.01,{ }^{\star * *} \mathrm{P}<0.001$.
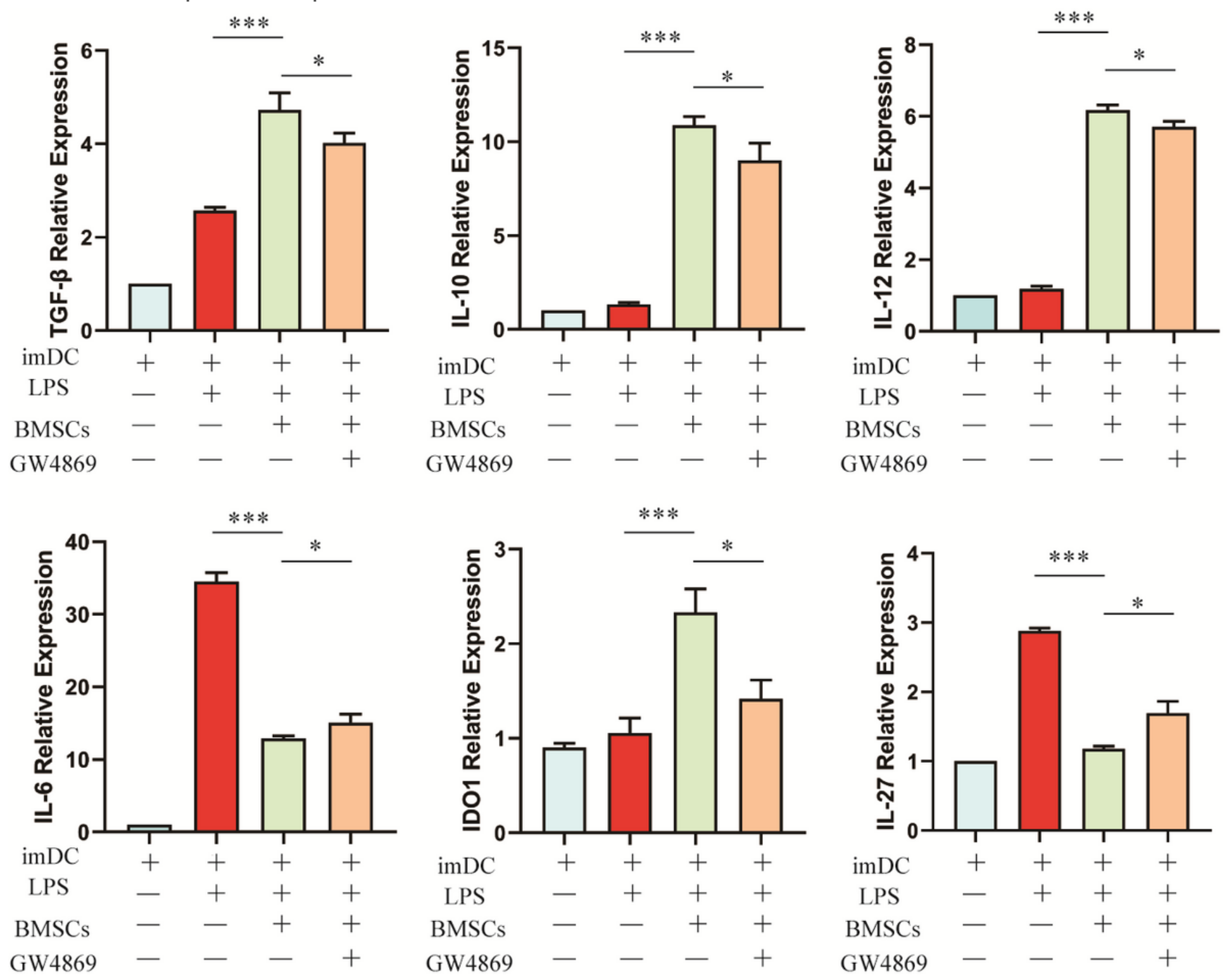

Figure 4
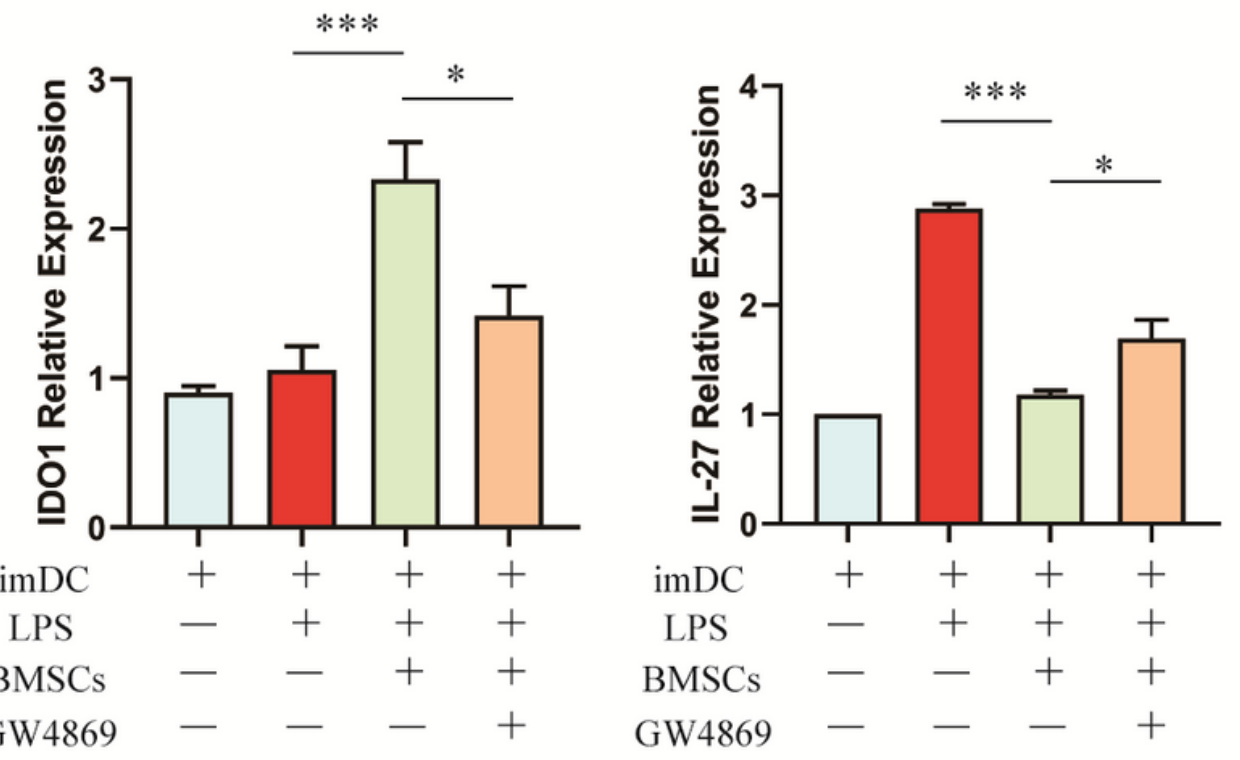

Quantitative RT-PCR analysis at 48h after co-cuthre showed the expression levels of genes of cytokines associated with immune activity (IL-6 and IL-27) and immune regulation (TGF- $\beta$, IL-10, IL-12 and IDO). *P $<0.05, * \star P<0.01, * \star * P<0.001$. 
A

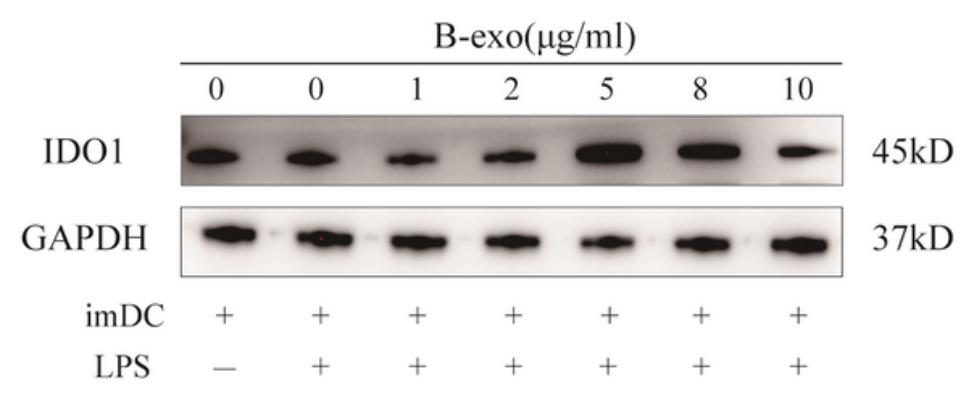

$\mathrm{C}$
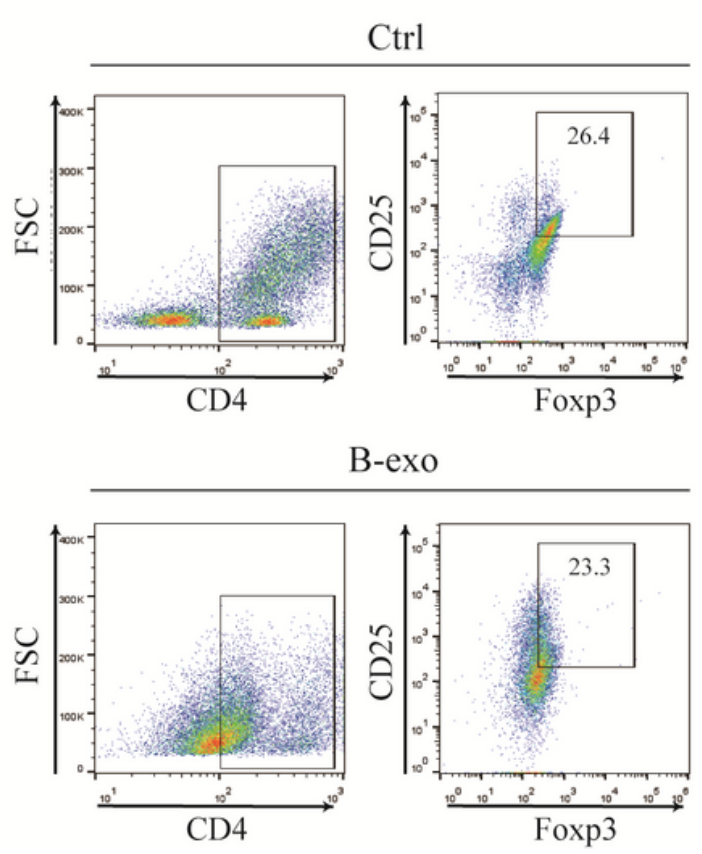

B

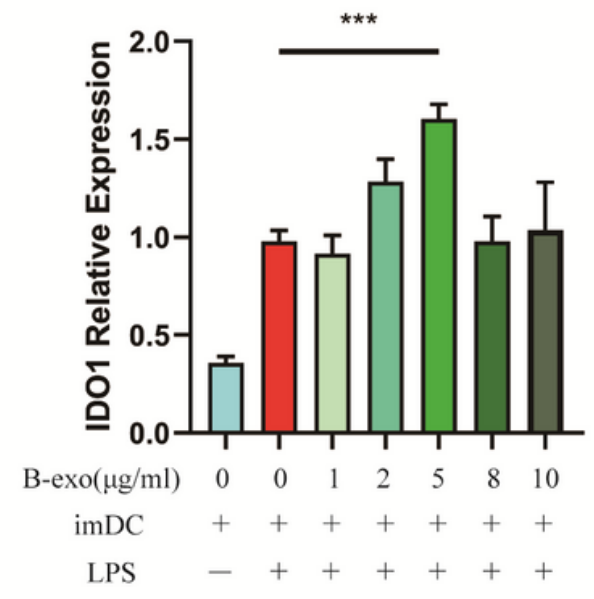

$\mathrm{D}$
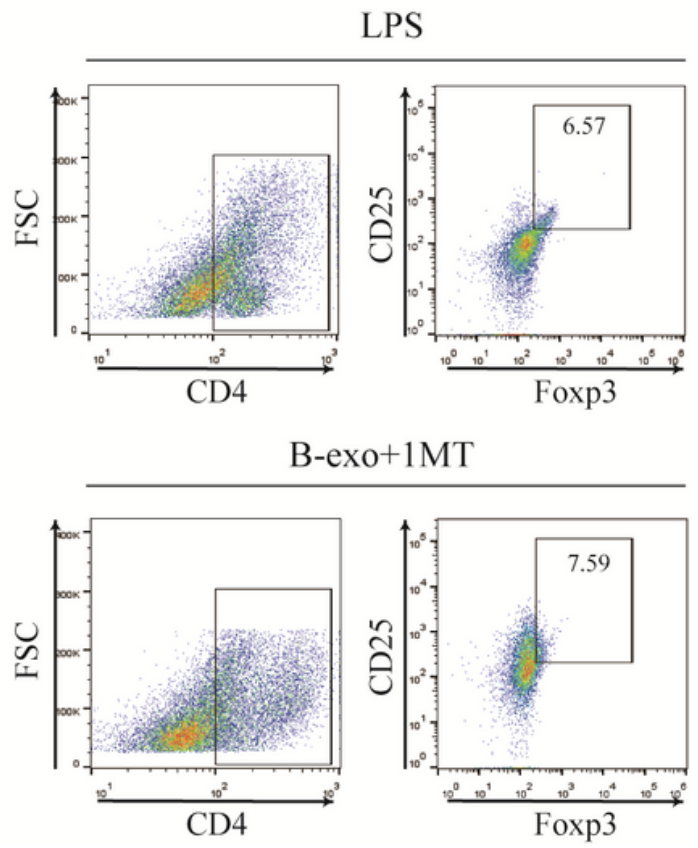

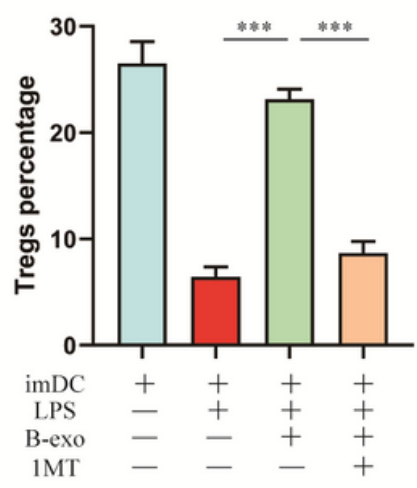

\section{Figure 5}

Effect of IDO on B-exos-exposed DCs A: The protein levels of IDO in DCs were changed by exposure with $B$-exo in different concentrations; $B$ : The gene expression levels of IDO in DCs were changed by exposure with B-exo in different concentrations. C: The percentage of CD4+Foxp3+T cells was analysed by flow cytometry after naïve CD4+ T was co-cultured with DCs; D: Cumulative data showed the percentage of CD4+Foxp3+T cells. ${ }^{\star} \mathrm{P}<0.05,{ }^{\star *} \mathrm{P}<0.01,{ }^{\star \star *} \mathrm{P}<0.001$. 
A

A

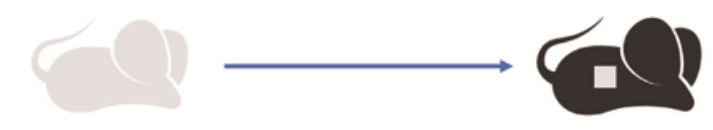

DCs $5 \times 10^{6}$ cell iv

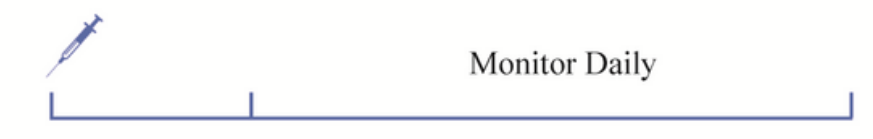

$\begin{array}{lll} & \text { POD0 } & \\ & \text { POD35 }\end{array}$

Skin Tx Remove the bandage
B

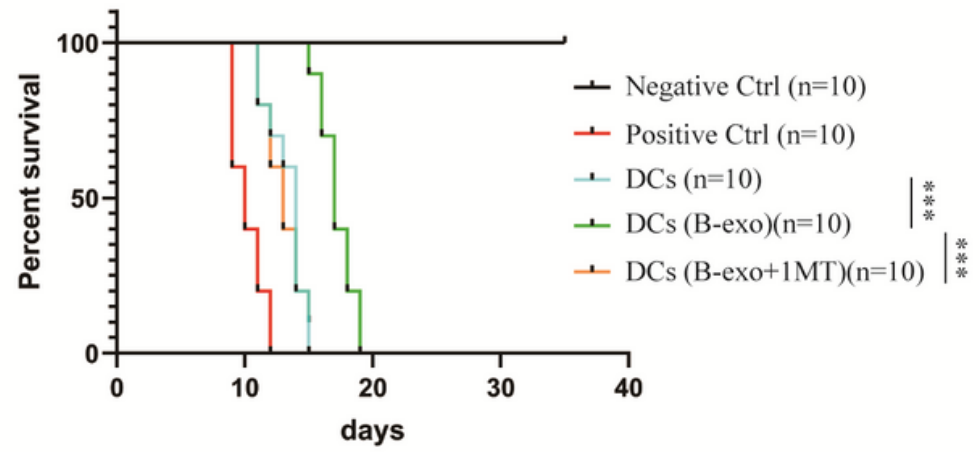

$\mathrm{C}$

POD12

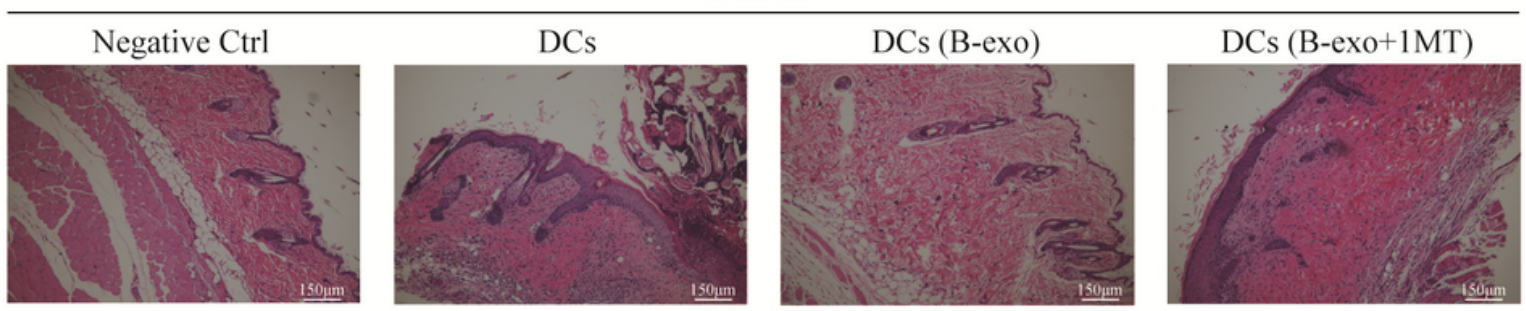

Figure 6

B-exos-exposed DCs enhanced allogeneic skin graft. A: Flow chart of mice skin transplantation surgery; B: Survival of skin grafts in different groups. Graft survival was compared by Kaplan-Meier analysis and the log-rank test $(n=10) ; C$ : Hematoxylin and Eosin Stain of skin grafts in different groups on POD12.

A

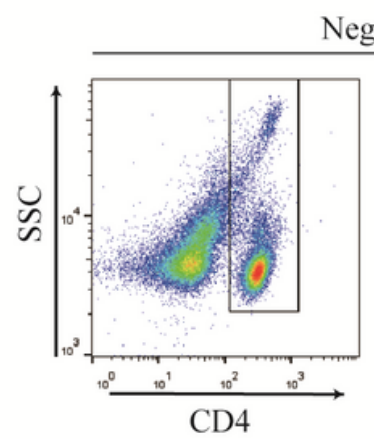

Negative Ctrl (POD12)
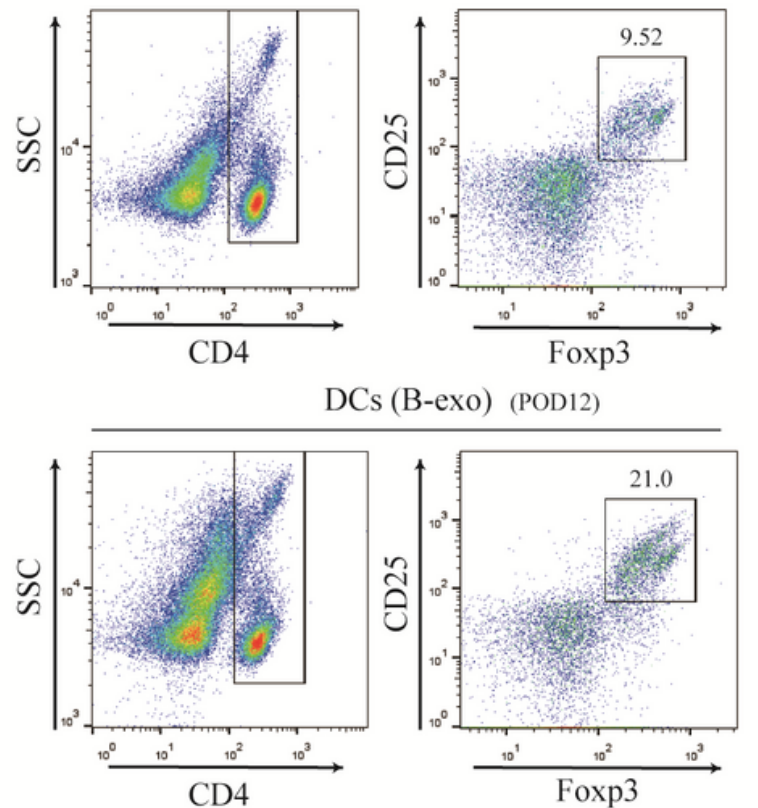

DCs (POD12)
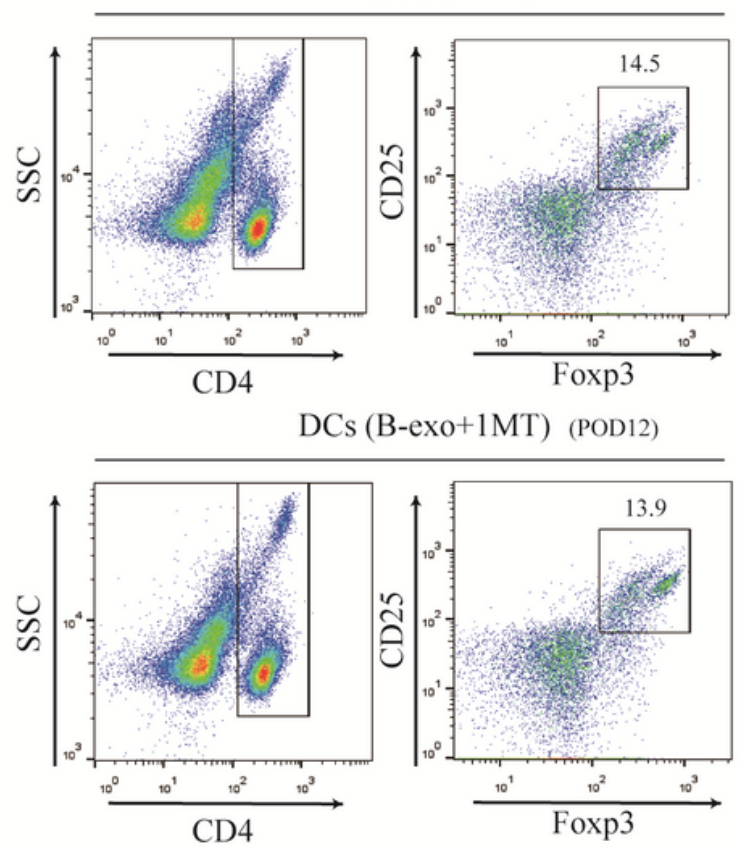

$\mathrm{B}$
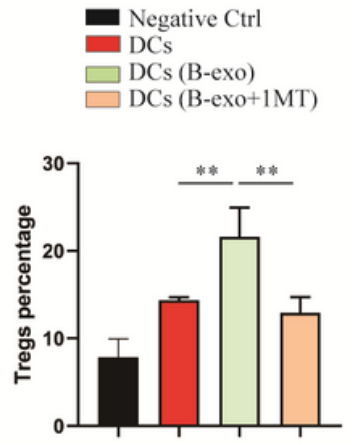

POD12

Figure 7 
$A$ : The percentage of $C D 4+F o x p 3+T$ cells in spleen from recepients was analysed by flow cytometry; $B$ : Cumulative data showed the percentage of $\mathrm{CD} 4+\mathrm{Foxp} 3+\mathrm{T}$ cells in spleen from recepients. Data represent mean \pm SEM $(n=3) .{ }^{*} P<0.05,{ }^{\star *} P<0.01, * \star * P<0.001$.

\section{Supplementary Files}

This is a list of supplementary files associated with this preprint. Click to download.

- SupplementaryFigure1.tif 